\title{
O impacto do tratamento do cliente com tuberculose internado em uma unidade hospitalar: uma revisão de literatura
}

The impact of treating clients with tuberculosis admitted to a hospital unit: a literature review

\author{
Solange da Cunha Ferreira* \\ Mestranda do Curso de Gestão e Saúde Pública, Universidade Columbia, Del Paraguai; Rio de Janeiro, Brasil. *Autor para \\ correspondência.E-mail: solferreira1306@gmail.com
}

\begin{abstract}
Resumo: Introdução: A tuberculose apesar de ser tratável e curável pode evoluir para a forma grave que requer internação do cliente para uma recuperação eficaz, principalmente quando é evidenciado o diagnóstico de doenças compulsórias. $\mathrm{O}$ estudo tem como objetivo discutir na literatura o isolamento respiratório relacionado ao cliente com tuberculose no ambiente hospitalar. Trata-se de um estudo de revisão de literatura, com abordagem qualitativa, que teve como questão de pesquisa: Como a literatura discute o isolamento respiratório relacionado ao cliente com tuberculose no ambiente hospitalar. Os estudos analisados permitiram a identificação da unidade temática "O isolamento respiratório relacionado ao cliente com tuberculose no ambiente hospitalar" e as seguintes categorias: $1 \mathrm{~A}$ visão dos custos do cliente com tuberculose no ambiente hospitalar; $2 \mathrm{O}$ cuidado ao cliente com tuberculose no ambiente hospitalar. Revisão e discussão: Para delimitar a busca, utilizou-se os critérios de inclusão: artigos disponíveis em português, no recorte temporal de 2014 a 2018, no site da Biblioteca Virtual em Saúde, com os descritores: Tuberculose; Insuficiência respiratória; Hospitalização e de 2012 a 2018 no Google Acadêmico, coma temática do estudo. Considerações finais: Evidenciou a importância da atenção básica, para que o paciente com tuberculose não complique, pois este no ambiente hospitalar aumenta os custos de internação, como também pode necessitar de tratamentos não convencionais para a doença, seja pela multirresistência ou pelo quadro agravado do indivíduo.
\end{abstract}

Palavras-chave: cliente, hospitalização, insuficiência respiratória, isolamento, tuberculose.

\begin{abstract}
Introduction: Despite being treatable and curable, tuberculosis can evolve to the severe form that requires hospitalization of the client for an effective recovery, especially when the diagnosis of compulsory diseases is evidenced. The study aims to discuss in the literature the respiratory isolation related to the client with tuberculosis in the hospital environment. This is a literature review study, with a qualitative approach, which had the following research question: How the literature discusses respiratory isolation related to the client with tuberculosis in the hospital environment. The analyzed studies allowed the identification of the thematic unit "Respiratory isolation related to the client with tuberculosis in the hospital environment" and the following categories: 1 The view of the cost of the client with tuberculosis in the hospital environment; 2 Care for clients with tuberculosis in the hospital environment. Review and discussion: To define the search, the inclusion criteria were used: articles available in Portuguese, in the time frame from 2014 to 2018, on the Virtual Health Library website, with the descriptors: Tuberculosis; Respiratory failure; Hospitalization and from 2012 to 2018 in Google Scholar, with the study them. Final considerations: It highlighted the importance of primary care, so that the patient with tuberculosis does not complicate, as this in the hospital environment increases hospitalization costs, as well as may require unconventional treatments for the disease, either due to the multidrug resistance or the individual's aggravated condition.
\end{abstract}

Keywords: client, hospitalization, respiratory failure, isolation, tuberculosis.

\section{Introdução}

A tuberculose é vista como problema de saúde pública principalmente na população de níveis econômicos baixos na maioria dos países, tanto que se estima um terço no mundo e tem como microbiota causadora o Mycobacterium tuberculosis, mas em território nacional a incidência de notificação ainda é alta na população brasileira (Souza Junior et al., 2018; Silva et al., 2012). 
As internações evitáveis são aquelas que não ocorreriam se a assistência à saúde fosse manejada com qualidade e em tempo oportuno no âmbito da Atenção Primária à Saúde (APS). A tuberculose está inserida, desde 2008, na lista brasileira das internações reconhecidas como evitáveis (Yamamura et al., 2016).

Dessa forma, a tuberculose normalmente é tratada na atenção básica, porém os casos mais graves requerem internação hospitalar e mesmo assim, a mortalidade nas instituições de saúde terciária é elevada por causa do estado de saúde que o indivíduo apresenta que geralmente, evidencia-se a dificuldade no acesso ao programa de tubérculo ou pela baixa capacidade resolutiva da rede de saúde (Silva et al., 2012; Yamamura et al., 2016).

Assim, a tuberculose, provoca mudanças negativas, como o medo e um forte estigma, o que provoca um afastamento e isolamento, por afetar as relações com as pessoas, e, além disso, infelizmente o preconceito em conexão ao medo afasta a comunidade que deixa, por muitas vezes, de prestar ajuda e solidariedade ao indivíduo (Araújo, 2017).

Embora a tuberculose apresente abrangência mundial, possui maior incidência em países emergentes, atingindo todas as categorias populacionais, entretanto, prevalece em pessoas do sexo masculinas e economicamente ativas. No Brasil, estima-se uma prevalência de 110.000 infectados, com cerca de 70.000 casos novos e 5.000 mortes anuais. Em decorrência disso, o Brasil ocupa a $17^{\mathrm{a}}$ posição entre os 22 países com maior carga de Tuberculose (Souza Junior et al., 2018).

Porém, existem as internações pela falta de resposta ao tratamento, cuja maioria não segue as recomendações e posologia do tratamento, gravando o seu estado de saúde, o que reflete na necessidade de hospitalização e muitos dos casos requerem a admissão na UTI (Silva et al.2014).

Dessa forma, o estudo objetivou discutir na literatura o isolamento respiratório relacionado ao cliente com tuberculose no ambiente hospitalar.

\section{Revisão}

Este estudo de revisão de literatura, tem o intuito de verificar as publicações relacionadas a temática do estudo, principalmente para evidenciar no campo científico o conhecimento (Souza et al., 2010), com abordagem qualitativa, que tem como questão de pesquisa: Como a literatura discute o isolamento respiratório relacionado ao cliente com tuberculose no ambiente hospitalar?

Para delimitar a inclusão dos estudos, utilizaram-se os seguintes critérios: artigos disponíveis em português, no recorte temporal de 2014 a 2018, no site da Biblioteca Virtual em Saúde (BVS), com o cruzamento dos descritores: Tuberculose; Insuficiência respiratória; Hospitalização.

Ressalto que os critérios de exclusão estabelecidos foram: indisponibilidade de acesso, publicações em mais de uma base de dados, resumo, textos na forma de projetos, em outros idiomas, fora do recorte temporal definido nos critérios de inclusão e todos os artigos que não são articulados a temática.

Quadro 1. Cruzamento dos descritores na BVS.

\begin{tabular}{|c|c|c|c|c|c|c|c|c|}
\hline Descritores & Total & Filtro & Seleção & $\mathbf{2 0 1 4}$ & $\mathbf{2 0 1 5}$ & $\mathbf{2 0 1 6}$ & $\mathbf{2 0 1 7}$ & $\mathbf{2 0 1 8}$ \\
\hline Tuberculose e Insuficiência respiratória e Hospitalização & 14 & 0 & 0 & 0 & 0 & 0 & 0 & 0 \\
\hline Tuberculose e Insuficiência respiratória & 561 & 01 & 0 & 0 & 0 & 0 & 0 & 0 \\
\hline Tuberculose e Hospitalização & 1151 & 10 & 05 & 01 & 01 & 01 & 01 & 01 \\
\hline Insuficiência respiratória e Hospitalização. & 415 & 04 & 0 & 0 & 0 & 0 & 0 & 0 \\
\hline Total & 2141 & 15 & 05 & 01 & 01 & 01 & 01 & 01 \\
\hline
\end{tabular}

Ao utilizar os descritores, Tuberculose e Insuficiência respiratória e Hospitalização, obtive-se o total de 14, com os critérios de inclusão não se selecionou estudo. Já cruzando Tuberculose e Insuficiência respiratória, evidenciou-se 561, ao filtrar obtive-se 01 artigo que não foi escolhido.

Selecionado 05, nos anos de 2014 (01); 2015 (01); 2016 (01); 2017 (01) e 2018 (01).

Com a busca a cima, não se encontrou um número expressivo de artigos, o que nos impulsionou a realizar uma busca isolada dos descritores. Os descritores Tuberculose e Hospitalização captou-se 1.151, com o filtro 10, dos quais 05 artigos foram selecionados. Mas, ao cruzar Insuficiência respiratória e Hospitalização, tevese 415, ao filtrar 04 foram a amostra, em que não foi incluído estudos. Dessa forma, o estudo evidenciou 2.141 produções, com 15 que atenderam aos critérios de inclusão, dos quais foram da pesquisa. 
Quadro 2. Descritores Isolados na BVS.

\begin{tabular}{|c|c|c|c|c|c|c|c|c|}
\hline Descritores & Total & Filtro & Seleção & $\mathbf{2 0 1 4}$ & $\mathbf{2 0 1 5}$ & $\mathbf{2 0 1 6}$ & $\mathbf{2 0 1 7}$ & $\mathbf{2 0 1 8}$ \\
\hline Tuberculose & 142.649 & 525 & 0 & 0 & 0 & 0 & 0 & 0 \\
\hline Insuficiência respiratória & 37.399 & 120 & 0 & 0 & 0 & 0 & 0 & 0 \\
\hline Hospitalização & 108.036 & 917 & 0 & 0 & 0 & 0 & 0 & 0 \\
\hline Total & 288.084 & 1.616 & 0 & 0 & 0 & 0 & 0 & 0 \\
\hline
\end{tabular}

Como pode de ser observado, ao utilizar os descritores, o estudo evidenciou 288.084 produções, com 1.616 que atenderam aos critérios de inclusão, mas não encontramos artigos novos, apenas os que já tinham sido selecionados estudos.

Dessa forma, realizou-se uma busca no Google Acadêmico, que se utilizou os seguintes critérios: produções disponíveis em português, no recorte temporal de 2014 a 2018, com o título da pesquisa.

Com a busca no Google Acadêmico, captou-se apenas 02 estudos envolvidos com a temática, os que não foram selecionados eram relacionados a outras patologias e a tuberculose na atenção básica.

Quadro 3 Busca pela temática no Google Acadêmico.

\begin{tabular}{|c|c|c|c|c|c|c|c|c|c|c|}
\hline Descritores & Total & Filtro & Seleção & 2012 & 2013 & 2014 & 2015 & 2016 & 2017 & 2018 \\
\hline $\begin{array}{l}\text { Isolamento respiratório frente ao cliente com } \\
\text { tuberculose internado em uma hospitalar }\end{array}$ & 656 & 225 & 01 & 01 & 0 & 0 & 0 & 0 & 01 & 0 \\
\hline
\end{tabular}

Quadro 4. Artigos selecionados no estudo.

\begin{tabular}{|c|c|c|c|c|c|}
\hline Título & Objetivos & Periódicos & Ano & Autores & $\begin{array}{c}\text { Tipo de } \\
\text { documento }\end{array}$ \\
\hline $\begin{array}{l}\text { Internações hospitalares e } \\
\text { impacto financeiro por } \\
\text { tuberculose pulmonar na } \\
\text { Bahia, Brasil }\end{array}$ & $\begin{array}{c}\text { descrever os casos de internações } \\
\text { hospitalares e impacto financeiro da } \\
\text { tuberculose pulmonar no estado da } \\
\text { Bahia, Brasil, entre o período de } 2012 \\
\text { e } 2016\end{array}$ & $\begin{array}{c}\text { Enfermaría Actual } \\
\text { de Costa Rica }\end{array}$ & 2018 & $\begin{array}{l}\text { Souza Júnior } \\
\text { et al. }\end{array}$ & $\begin{array}{l}\text { Artigo } \\
\text { Original }\end{array}$ \\
\hline $\begin{array}{l}\text { Vulnerabilidade e direitos } \\
\text { humanos na compreensão de } \\
\text { trajetórias de internação por } \\
\text { tuberculose }\end{array}$ & $\begin{array}{l}\text { compreender como desigualdades e } \\
\text { violações de direitos humanos se } \\
\text { articulam em trajetórias de } \\
\text { vulnerabilidade à internação por } \\
\text { tuberculose } \\
\end{array}$ & Saúde soc. & 2017 & $\begin{array}{l}\text { Maffacciolli } \\
\text { et al. }\end{array}$ & $\begin{array}{l}\text { Artigo } \\
\text { Original }\end{array}$ \\
\hline $\begin{array}{l}\text { A gestão do atendimento aos } \\
\text { pacientes em tratamento de } \\
\text { tuberculose pulmonar: a } \\
\text { situação em duas unidades } \\
\text { hospitalares de Maceió/AL }\end{array}$ & $\begin{array}{l}\text { compreender de que forma a equipe } \\
\text { multiprofissional da Unidade } \\
\text { Hospitalar de Saúde percebem a } \\
\text { Gestão do atendimento a pacientes } \\
\text { em tratamento de tuberculose na } \\
\text { cidade de Maceió/AL }\end{array}$ & $\begin{array}{l}\text { Universidade } \\
\text { Lusófona de } \\
\text { Humanidades e } \\
\text { Tecnologias }\end{array}$ & 2017 & Araújo & $\begin{array}{l}\text { Dissertação } \\
\text { de Mestrado }\end{array}$ \\
\hline $\begin{array}{l}\text { Análise espacial das } \\
\text { internações evitáveis por } \\
\text { tuberculose em Ribeirão Preto, } \\
\text { SP (2006-2012) }\end{array}$ & $\begin{array}{l}\text { Descrever a distribuição espacial dos } \\
\text { casos de internações evitáveis por } \\
\text { tuberculose no município de Ribeirão } \\
\text { Preto, SP, e identificar aglomerados } \\
\text { espaciais e espaço-temporais de risco } \\
\text { para a ocorrência desses eventos }\end{array}$ & Rev. Saúde Pública & 2016 & $\begin{array}{c}\text { Yamamura et } \\
\text { al. }\end{array}$ & $\begin{array}{l}\text { Artigo } \\
\text { Original }\end{array}$ \\
\hline $\begin{array}{l}\text { O desafio do tratamento da } \\
\text { tuberculose extensivamente } \\
\text { resistente em um hospital de } \\
\text { referência no estado de São } \\
\text { Paulo: um relato de três casos } \\
\end{array}$ & $\begin{array}{c}\text { mostrar a evolução no período de } 1 \\
\text { ano de três pacientes com TB-XDR } \\
\text { internados no Hospital Nestor } \\
\text { Goulart Reis }\end{array}$ & J. bras. pneumol & 2015 & Arbex et al. & $\begin{array}{l}\text { Relato de } \\
\text { Caso }\end{array}$ \\
\hline $\begin{array}{c}\text { Tuberculose em pacientes } \\
\text { hospitalizados: características } \\
\text { clínicas dos pacientes que } \\
\text { iniciaram tratamento nas } \\
\text { primeiras } 24 \mathrm{~h} \text { de permanência } \\
\text { hospitalar }\end{array}$ & $\begin{array}{l}\text { Comparar as características clínicas e } \\
\text { os desfechos de pacientes } \\
\text { hospitalizados por tuberculose que } \\
\text { iniciaram tratamento nas primeiras } \\
24 \text { h de permanência hospitalar com } \\
\text { as daqueles que iniciaram tratamento } \\
\text { após } 24 \mathrm{~h}\end{array}$ & J. bras. pneumol & 2014 & Silva et al. & $\begin{array}{l}\text { Artigo } \\
\text { Original }\end{array}$ \\
\hline $\begin{array}{c}\text { Tuberculose grave com } \\
\text { necessidade de internação em } \\
\text { UTI }\end{array}$ & $\begin{array}{l}\text { fazer uma revisão dos aspectos } \\
\text { relacionados à tuberculose em } \\
\text { pacientes em UTIs e suas } \\
\text { peculiaridades }\end{array}$ & J. bras. pneumol & 2012 & Silva et al. & $\begin{array}{l}\text { Artigo } \\
\text { Original }\end{array}$ \\
\hline
\end{tabular}


Ao recorte temporal de 2014 a 2018, foi evidenciado 06 artigos em português, relacionado à temática do estudo, destes percebe-se as produções em 2012 (01); 2014 (01); 2015 (01); 2016 (01); 2017 (02) e 2018 (01).

As revistas evidenciadas dos artigos coletados foram: Enfermería Actual de Costa Rica; J. bras. pneumol.; Rev. Saúde Pública; Saúde soc. Houve apenas a Universidade Lusófona de Humanidades e Tecnologias. Sendo assim, o estudo foi construído com 06 artigos e 01 dissertação de mestrado.

Após os processamentos dos dados, consistiu a interpretação dos resultados relacionados a questão de pesquisa, em que foi realizado a análise seguindo os passos da análise temática de Bandin descrita por Minayo (2010), que se divide em três etapas.

A primeira etapa foi realizada a leitura de todos os artigos, para a impregnação do conteúdo permitindo a constituição do corpus, o que valida a abordagem qualitativa. Assim, foi possível delimitar a compreensão dos textos, para evidenciar as unidades de registros, pois a partir as partes que se identificam com o estudo do material tornou possível à formação das unidades temática, em que codificamos e utilizamos os conceitos teóricos levantados para a orientação da análise na etapa.

Na segunda etapa, houve a exploração do material, para encontrar as unidades de registro pelas expressões e palavras significativas, para classificar e agregar os dados no alcance do núcleo de compreensão do texto de forma organizada e sistemática.

Na Terceira Etapa, com os dados da análise, foi possível articular o referencial teórico, o que fez emergir a identificação da unidade temática "O isolamento respiratório relacionado ao cliente com tuberculose no ambiente hospitalar" e as seguintes categorias: 1 A visão dos custos do cliente com tuberculose no ambiente hospitalar; $2 \mathrm{O}$ cuidado ao cliente com tuberculose no ambiente hospitalar.

A análise dos dados foi realizada, baseada nos artigos selecionados, em que foi possível observar, contar e somar, descrever e qualificar os dados, para aglomerar o conhecimento produzido através da temática nessa revisão (Souza et al., 2010; Soares et al., 2014).

\section{Categorias1: A visão dos custos do cliente com tuberculose no ambiente hospitalar}

$\mathrm{Na}$ hospitalização do paciente há deslumbres voltados para os fatores de complicações da doença, que pode acontecer pelos aspectos biológicos imunológicos, manifestações comportamentais pela interfase cultural e marcadores socioeconômicos do indivíduo pela falta de informações (Maffacciolli et al., 2017; Yamamura et al., 2016).

As medidas de controle de transmissão da tuberculose podem ser divididas em três grupos distintos: medidas administrativas, em relação a investigação, diagnóstico, tratamento precoces, como também o isolamento dos pacientes suspeitos; medidas ambientais, reflete em quartos de isolamento com pressão negativa e uso de filtro high efficiency particulate air e medidas de proteção respiratória, com o uso de respiradores N95 pela equipe de saúde (Silva et al., 2012; Araújo, 2017).

Nessa perspectiva, o alto uso de recursos financeiros na prestação da assistência a nível hospitalar pelo SUS reflete a indispensabilidade de serviços de amparo direcionado à TP. Desse modo, a internação, o período de permanência, o atributo de exames de imagem e o Tratamento Diretamente Observado (TDO) resultam em um abalo orçamentário de grande expressividade no sistema de saúde (Souza Junior et al., 2018).

Assim, no ambiente hospitalar, com os pacientes suspeitos deve ser obrigatório a pesquisa de BAAR e cultura das secreções respiratórias e devem também serem colocados em quartos individuais com pressão negativa e a precaução respiratória, mas em pacientes em ventilação mecânica utiliza-se um sistema fechado para aspiração endotraqueal e filtro bacteriano no circuito expiratório (Maffacciolli et al., 2017; Silva et al., 2014).

As determinações de medidas assistencialistas devem ser utilizadas principalmente no meio hospitalar, mas devemos pensar na população para a redução da incidência de tuberculose e seus desdobramentos o que diminui os custos com internação (Souza Junior et al., 2018).

Por trazer impacto financeiro para os pacientes e também para os sistemas de saúde, um tratamento hospitalar excede até mil vezes o custo ambulatorial, apresentando, portanto, menor custo-efetividade em relação ao tratamento ambulatorial (Yamamura et al., 2016).

Dessa forma, os aspectos culturais são fatores que interferem no cotidiano, em que ao invés do indivíduo ser atendido na assistência primária prefere buscar o atendimento no hospital, por receio de identificação e discriminação, principalmente com o advento do HIV, o que colabora com a sobrecarrega dos serviços terciários de cuidado, o custo de internação e também nas taxas de morbimortalidade do quadro mediante a não realização do tratamento de tuberculose (Souza Junior et al., 2018; Araújo, 2017).

Estudos têm evidenciado a existência de carac $\neg$ terísticas comuns nos históricos de pessoas hos $\neg$ pitalizadas para tratar tuberculose. Dentre essas características, destacam-se: maior prevalência de pacientes do sexo masculino; predomínio da faixa etária entre 30 e 59 anos; uso prejudicial de álcool, tabaco e outras drogas; 
HIV/aids como principal comorbidade; histórico de prisão; ausência de mo-radia fixa; desemprego; rupturas nos laços afetivos e falta de apoio da família; reincidência da doença e necessidade de retratamento; baixa escolaridade e renda inferior a um salário mínimo (Maffacciolli et al., 2017).

Desta forma, por medida de proteção em risco ocupacional aos profissionais é oferecido o uso da máscara com o filtro N-95 em casos de isolamento de pacientes com tuberculose pulmonar, é um procedimento que deverá ser utilizado na assistência de enfermagem, em fase de transmissibilidade, pois alguns autores apontaram que os trabalhadores da saúde são grupos mais vulneráveis para adquirir a enfermidade (Araújo, 2017).

Contudo, estudos ainda recomendam que a instituição faça um inquérito tuberculínico nos profissionais de saúde que trabalham em áreas de risco, para avaliar por ocasião de sua admissão, e testagens periódicas para a identificação dos conversores, para a infecção latente, tanto, que devem ser orientados de acordo com os critérios de consenso (Maffacciolli et al., 2017; Silva et al., 2014).

\section{Categorias 2: 0 cuidado ao cliente com tuberculose no ambiente hospitalar}

O atendimento ao paciente com tuberculose é multiprofissional, no que tange a importância da atuação simultânea oferta a assistência integral, pela exigência do conhecimento especializado com abrangência coletiva e individual do processo saúde doença (Araújo, 2017).

No Brasil, o tratamento da tuber`culose multirresistente é padronizado pelo Ministério da Saúde. A OMS recomenda o tratamento da tuber $\neg$ culose multirresistente em duas fases: intensiva e de manutenção. A fase intensiva, com duração de 8 meses, deve incluir no mínimo quatro drogas potencialmente efetivas: uma droga injetável, uma fluoroquinolona, uma droga oral e um fármaco de reforço. $\mathrm{Na}$ fase de manutenção, retira-se a droga injetável, e as outras drogas devem ser mantidas por 12 meses após a negativação da cultura do escarro (Arbex et al., 2015).

A insuficiência respiratória decorrente de tuberculose era relatada como uma das causas de admissões no ambiente hospitalar, que necessitavam de internação em UTI, cujos sintomas mais comumente apresentados eram febre, sudorese noturna, emagrecimento, tosse e dispneia, em que os fatores associados ao desenvolvimento de insuficiência respiratória estava relacionada a pneumonia, que poderia ser seguida de sepse, história de DPOC ou de tratamento irregular para tuberculose (Maffacciolli et al., 2017). Trata-se de uma revisão de literatura que, segundo Gil (2008), foi realizado a partir do levantamento de referências teóricas já analisadas e publicadas, por meios escritos e eletrônicos, como livros, artigos científicos, páginas de internet.

\section{Discussão}

Em um estudo, foi observada a necessidade de internação em UTI em pacientes que apresentavam tuberculose miliar/disseminada e pneumonia tuberculosa, que os achados laboratoriais foram encontrados nos exames anemia, leucopenia, leucocitose e hipoalbuminemia (Arbex et al., 2015; Silva et al 2012).

Os pacientes com tuberculose que o padrão associado era de dano alveolar difuso, necessitaram de cuidados intensivos, que alguns evoluíram para síndrome da angústia respiratória aguda, porém apesar das características clínicas e radiológicas, houve pacientes cujos achados histopatológicos apontavam para a broncopneumonia tuberculosa (Maffacciolli et al., 2017).

Como a cultura para micobactérias demora 6-8 semanas, o tratamento dos pacientes internados em UTIs raramente eram baseados em resultados de culturas, pois a obtenção de material para análise poderia ter dificuldades principalmente em pacientes com tuberculose extrapulmonar e em ventilação mecânica onde os parâmetros não permitiam a broncoscopia, por isso, que se sugere o início das medicações antes do resultado dos exames diagnósticos, pois o atraso no início do tratamento pode levar a óbito (Silva et al 2014; Yamamura et al., 2016; Arbex et al., 2015).

A radiografia de tórax não contribui para o diagnóstico de doença em atividade, como nos casos de alterações parenquimatosas mínimas e na diferenciação de lesões antigas fibróticas das lesões características de disseminação broncogênica (Maffacciolli et al., 2017).

Os pacientes com tuberculose internada em UTIs podem desenvolver outras complicações, tais como pneumonia associada à ventilação mecânica, falência de múltiplos órgãos, choque séptico, insuficiência renal aguda, coagulação intravascular disseminada e sangramento digestivo (Araújo, 2017). 
A mortalidade intra-hospitalar dos pacientes com tuberculose permanece alta, particularmente entre os pacientes que necessitam admissão em UTI. A insuficiência respiratória aguda causada por tuberculose e com necessidade de VM tem sido associada com taxas de mortalidade (Arbex et al., 2015; Silva et al., 2014).

Mesmo após a alta hospitalar, a mortalidade mantém-se elevada. Em uma série de casos com pacientes hospitalizados com tuberculose e insuficiência respiratória, a mortalidade foi detectada nos três meses seguintes à alta hospitalar (Yamamura et al., 2016).

Em alguns estudos, têm sido relatados fatores que podem contribuir para a mortalidade entre pacientes com tuberculose criticamente doentes como no contexto de infecção pelo HIV, tem sido reconhecida como um importante fator preditor de óbito, como também em outros fatores que podem influenciar as taxas de mortalidade na presença de doença fibrocavitária extensa e de consolidações na radiografia de tórax (Arbex et al., 2015; Silva et al., 2012).

\section{Considerações finais}

O estudo apontou, para um tratamento multiprofissional, cujos pacientes com tuberculose no momento de complicação da doença têm necessidade de internação no ambiente hospitalar e em alguns casos até em UTI.

Na maioria dos casos apresentam insuficiência respiratória com os sinais e sintomas aflorados, porém alguns pacientes são internados sem o diagnóstico o que reflete no não tratamento da doença, o que agrava a situação, o que pode levar a morte.

Apesar do diagnóstico e tratamento da tuberculose ser realizado na atenção básica, os casos particularmente complicados até pela multirresistência, pode acontecer na interpretação errônea no exame de imagem, pela dificuldade de obtenção da secreção à análise de micobactérias e à indisponibilidade de cultura pelo estado do paciente e demora dos resultados, o que retarda o tratamento.

Um dos pontos evidenciados na pesquisa, está referente ao alto custo no ambiente hospitalar, o que reforça a importância do diagnóstico e tratamento da tuberculose na atenção básica, que para a economia do país é extremamente vantajoso, mas para tal, campanhas devem ser realizadas na população e o alerta dos sinais, sintomas da doença para irem ao serviço primário e não no terciário.

Assim, o uso de esquemas medicamentosos diferenciados é importante, principalmente por causa da diminuição do metabolismo hepático e concentração plasmática das drogas antituberculose, o que interfere direta ou indiretamente no plano terapêutico dos profissionais de saúde para o paciente.

A atenção hospitalar é importante para o prognóstico dos pacientes que houve complicação da doença, não seja tão negativo ou até mesmo apareça nas taxas de mortalidade.

Mediante ao escrito, cabe reforçar que as ações contra a tuberculose devem ser realizadas na rede pública, cujas emergências hospitalares, na classificação de risco devem redirecionar este paciente para o tratamento adequado na atenção básica, porém, os indivíduos com risco de insuficiência respiratória devem ser observados e cabe a equipe multidisciplinar a necessidade de terapia intensiva.

\section{Referências}

Arbex, M. A., Siqueira, H. R., D’Ambrosio, L., \& Migliori, G. B. 2015. The challenge of managing extensively drug-resistant tuberculosis at a referral hospital in the state of São Paulo, Brazil: a report of three cases. Jornal Brasileiro de Pneumologia, 41(6), 554-559.

Araújo, L. M. A. A gestão do atendimento aos pacientes em tratamento de tuberculose pulmonar: a situação em duas unidades hospitalares de Maceió/AL. 2017. Dissertação (Mestrado em Gestão de Empresas no Curso de Mestrado em Gestão de Empresas), Universidade Lusófona de Humanidades e Tecnologias, Escola de Ciências Económicas e das Organizações.

Gil, A. C. 2008. Como elaborar projetos de pesquisa. São Paulo, SP: Atlas.

Maffacciolli, R.; Oliveira, D. L. L. C.; \& Brand, E. M. 2017. Vulnerabilidade e direitos humanos na compreensão de trajetórias de internação por tuberculose. Saúde e Sociedade, 26(1), 286-299.

Minayo, M. C. S. 2010. O desafio do conhecimento: Pesquisa Qualitativa em Saúde (12a ed.). São Paulo, SP: Hucitec-Abrasco.

Silva, D. R., Silva, L. P., \& Dalcin, P. T. R. 2014. Tuberculose em pacientes hospitalizados: características clínicas dos pacientes que iniciaram tratamento nas primeiras $24 \mathrm{~h}$ de permanência hospitalar. Jornal Brasileiro de Pneumologia, 40(3), 279-285. 
Silva, D. R., Gazzana, M. B., \& Dalcin, P. T. R. 2012. Tuberculose grave com necessidade de internação em UTI. Jornal Brasileiro de Pneumologia, 38(3), 386-394.

Soares, C. B., Hoga, L. A. K., Peduzzi, M., Sangaleti, C., Yonekura, T., \& Silva, D. R. A. D. 2014. Revisão integrativa: conceitos e métodos utilizados na enfermagem. Revista da Escola de Enfermagem da USP, 48(2), 335-345.

Souza, M. T.; Silva, M. D.; \& Carvalho, R. 2010 Revisão integrativa: o que é e como fazer. Einstein, 8(1), 102-06.

Souza Junior, E. V., Nunes, G. A., Cru, D. P., Boery, E. N., \& Boery, R. N. S. O. 2018. Internações hospitalares e impacto financeiro por tuberculose pulmonar na Bahia, Brasil. Enfermería Actual de Costa Rica, 35, 38-51.

Yamamura, M., Freitas, I. M., Neto, M. S., Neto, F. C., Popolin, M. A. P., Arroyo, L. H., Rodriguez, L. B. B., Crispim, J. A., \& Arcêncio, R. A. 2016. Análise espacial das internações evitáveis por tuberculose em Ribeirão Preto, SP (2006-2012). Revista de Saúde Pública, 50(20), 1-11.

\section{Minicurrículo}

Solange da Cunha Ferreira. Mestrado em progresso em Gestão da Saúde Pública pela Universid Columbia Del Paraguay. Com pós-graduação em Lato Sensu em Saúde Pública e Bacharelado em Enfermagem pelo Centro Universitário Celso Lisboa (2013), Rio de Janeiro- Brasil. Atualmente, funcionária pública, do Hospital Universitário Clementino Fraga Filho (HUCFF), Rio de Janeiro- Brasil.

\footnotetext{
Como citar: Ferreira, S.C. 2020. O impacto do tratamento do cliente com tuberculose internado em uma unidade hospitalar: uma revisão de literatura. Pubsaúde, 4, a064. DOI: https://dx.doi.org/10.31533/pubsaude4.a064

Recebido: 14 ago. 2020.

Revisado e aceito: 25 ago. 2020.

Conflito de interesse: os autores declaram não haver conflito de interesse.

Licenciamento: Este artigo é publicado na modalidade Acesso Aberto sob a licença Creative Commons Atribuição 4.0 (CC-BY 4.0).
} 